\title{
PROGRAMAS DE CAPACITACIÓN PARA EL FORTALECIMIENTO DE CAPACIDADES EN INFORMÁTICA EN ENFERMERÍA: UNA PERSPECTIVA PERUANA
}

\author{
TRAINING PROGRAMS FOR CAPACITY-BUILDING IN NURSING \\ INFORMATICS: A PERUVIAN PERSPECTIVE
}

\author{
PROGRAMAS DE TREINAMENTO DE CAPACITAÇÃO EM \\ INFORMÁTICA EM ENFERMAGEM: UMA PERSPECTIVA PERUANA
}

\author{
Walter H. Curioso* \\ Elsa Oscuvilca-Tapia**
}

\begin{abstract}
RESUMEN
El personal de enfermería tiene el gran desafío de aprovechar los nuevos recursos tecnológicos para llevar a cabo su trabajo de manera más eficiente y efectiva. La informática en enfermería se ha descrito como una competencia esencial para los profesionales de enfermería en varios países. En Perú, el personal de enfermería es predominantemente femenino y representa la mayor fuerza de trabajo en el sistema de salud. Este artículo analiza los desafíos para los programas de capacitación para el desarrollo de capacidades en informática en enfermería en el Perú. En general, la literatura publicada sobre los programas de capacitación en informática en enfermería en el Perú es escasa. Solo una de cada cuatro universidades peruanas ofrece cursos relacionados con informática en salud en los programas académicos de pregrado en enfermería. En el 2020, el Ministerio de Salud del Perú definió las competencias básicas de enfermería para gestionar las tecnologías de información y comunicación, y los sistemas de información de salud, incluida la telemedicina. Sin embargo, la informática en enfermería aún no se ha extendido ampliamente en el Perú debido a desafíos organizacionales, de recursos, y factores culturales y socio-técnicos. Además, las pandemias, como el COVID-19, requieren una acción urgente para transformar la prestación de atención de la salud, para expandir los programas e iniciativas de teleenfermería y la educación a distancia en el Perú. Es esencial promover programas de investigación y desarrollo de capacidades en informática en enfermería en el Perú y fomentar redes de colaboración global entre instituciones públicas y privadas.
\end{abstract}

Palabras clave: Enfermería; Informática; Capacitación profesional; Educación; Fortalecimiento institucional, Perú.

\footnotetext{
*Médico cirujano, PhD en Informática Biomédica. Universidad Continental, Perú. ORCID: https://orcid.org/0000-0002-50685357 Email: wcurioso@continental.edu.pe Autor de correspondencia.

**Enfermera, Doctora en Salud Pública. Universidad Nacional José Faustino Sánchez Carrión, Perú. ORCID: https://orcid.org/ 0000-0003-0586-875X Email: elsaoscuvilca@gmail.com
} 


\begin{abstract}
Nursing personnel has to face the great challenge of taking advantage of new technological resources to carry out their work in a more efficient and effective way. Nursing informatics has been described as an essential competence for nurse professionals in several countries. In Peru, nursing personnel is predominantly female and represents the largest workforce group in the healthcare system. This article discusses the challenges for capacity-building training programs in nursing informatics in Peru. In general, the published literature on nursing informatics training programs in Peru is rather scarce. Only one out of four Peruvian universities offer courses related to health informatics in undergraduate nursing academic programs. In 2020, the Peruvian Ministry of Health defined the core nursing competences to manage information and communication technologies, and health information systems, including telemedicine. However, nursing informatics has not yet been sufficiently developed in Peru due to organizational, material, cultural and sociotechnical challenges. Moreover, pandemics such as COVID-19 require urgent action to transform health care delivery, to expand telenursing and distance learning nursing programs and initiatives in Peru. Therefore, it is essential to promote research and capacity-building programs in nursing informatics in Peru and foster global collaborative networks between public and private institutions.
\end{abstract}

Key words: Nursing; Informatics; Professional training; Education; Capacity building; Peru.

\title{
RESUMO
}

O pessoal de enfermagem tem o grande desafio de aproveitar novos recursos tecnológicos para realizar seu trabalho de maneira mais eficiente e eficaz. A informática em enfermagem tem sido descrita como uma competência essencial para profissionais de enfermagem em vários países. No Peru, o pessoal de enfermagem é predominantemente feminino e representa o maior grupo de força de trabalho no sistema de saúde. Este artigo discute os desafios dos programas de treinamento de capacitaçáo em informática em enfermagem no Peru. Em geral, a literatura publicada sobre os programas de treinamento em informática em enfermagem no Peru é escassa. Apenas uma de cada quatro universidades peruanas oferece cursos relacionados à informática em saúde em programas acadêmicos de graduação em enfermagem. Em 2020, o Ministério da Saúde do Peru definiu as principais competências de enfermagem no gerenciamento de tecnologias da informação e comunicação e sistemas de informação em saúde, incluindo a telemedicina. No entanto, a informática em enfermagem ainda não foi desenvolvida amplamente no Peru devido a desafios organizacionais, físicos, e fatores culturais e sociotécnicos. Além disso, pandemias como a COVID-19 exigem uma ação urgente para transformar a prestação de serviços de saúde, para expandir os programas e iniciativas de telenfermagem e o ensino a distância no Peru. É essencial promover programas de pesquisa e desenvolvimento de capacidades em informática em enfermagem no Peru e promover redes globais de colaboração entre instituiçóes públicas e privadas.

Palavras-chave: Enfermagem; Informática; Capacitação profissional; Educação; Fortalecimento institucional; Peru.

Fecha de recepción: 22/09/2020

Fecha de aceptación: 24/01/2021

\section{INTRODUCCIÓN}

Según la Organización Mundial de la Salud, las enfermeras desempeñan un papel fundamental en la promoción de la salud, la prevención de enfermedades y la prestación de servicios de atención primaria y comunitaria ${ }^{(1)}$, y en ese sentido, las enfermeras se encuentran a la vanguardia del proceso de transformación del cuidado de la salud, sobre todo con los más vulnerables, en el contexto 
del logro de los objetivos de desarrollo sostenible a partir de la Agenda 2030 de las Naciones Unidas ${ }^{(2)}$.

El personal de enfermería hoy tiene un gran desafío: Aprovechar los nuevos recursos tecnológicos para realizar su trabajo de una manera más eficiente y eficaz. Es fundamental que los programas académicos de enfermería y las universidades revisen sus currículos alineados con los avances tecnológicos en salud digital, promoviendo el desarrollo de las habilidades digitales e informáticas necesarias para el desempeńo profesional, de acuerdo con las recomendaciones internacionales ${ }^{(3,4)}$.

La informática en enfermería es definida por la American Nurses Association (ANA) como una "especialidad que integra la ciencia de la enfermería con múltiples ciencias de la información y analíticas para identificar, definir, administrar y comunicar datos, información, conocimiento y sabiduría en la práctica de la enfermería"(5).

Además, la informática en enfermería se ha descrito como una competencia esencial para los profesionales de enfermería en países como los Estados Unidos de América y Canadá, mientras que en países como Nueva Zelanda, Inglaterra, Finlandia y Australia, la competencia de informática en enfermería varía considerablemente ${ }^{(6)}$.

Los programas de formación de informática en enfermería, en todo el mundo, varían entre países en relación con la duración del programa educativo, el lugar del programa educativo y el equilibrio entre la teoría y la práctica dentro del plan de estudios $^{(6)}$. Es esencial que los(as) enfermeros(as) y los(as) estudiantes de enfermería adquieran competencias para utilizar con éxito las tecnologías de la información y comunicación en su educación y práctica para beneficiar a los pacientes y mejorar su desempeńo. Por ello, este artículo analiza los desafíos de los programas de capacitación en informática en enfermería desde una perspectiva peruana.

\section{PROGRAMAS DE FORMACIÓN EN INFORMÁTICA EN ENFERMERÍA EN AMÉRICA LATINA}

Las competencias informáticas requeridas por los(as) enfermeros(as) a nivel mundial han aumentado sustancialmente en diversidad $y$ complejidad durante la última década ${ }^{(7)}$. Este mayor nivel de habilidad es indicativo de la necesidad de desarrollar y evaluar periódicamente las competencias en informática en enfermería en América Latina ${ }^{(8)}$. Para preparar mejor a los estudiantes de pre y posgrado, los educadores de enfermería deben estar debidamente capacitados en el uso de sistemas electrónicos de información en salud, manteniéndose al día con la literatura de enfermería, entre otras habilidades en informática en enfermería ${ }^{(9)}$. Sin embargo, en muchos países de América Latina, la recopilación de datos todavía se registra en formularios en papel y solo está relacionada con el encuentro del paciente ${ }^{(10)}$. Además, la falta de infraestructura adecuada, la conexión lenta a Internet y el bajo ancho de banda en las ciudades peruanas, las desigualdades en el acceso a la electricidad, el analfabetismo digital y la resistencia al cambio, son desafíos importantes para considerar $^{(9)}$.

Los países de América Latina han desarrollado una amplia gama de iniciativas de informática en enfermería, incluidos programas educativos y de fortalecimiento de capacidades en informática ${ }^{(11-16)}$. En la región, uno de los países con más experiencia en programas de educación en informática de enfermería es Brasil, que inició la informática en la profesión en el año 1985, cuando profesores de enfermería de la Universidad Federal de Rio Grande do Sul presentaron experiencias en el uso de computadoras para la enseñanza de enfermería ${ }^{(8)}$. Cinco años después se organizó una sesión de informática de enfermería en la Sociedad Brasileña de Informática en Salud. En 1991, el Simposio Interamericano de Informática en Enfermería fue organizado por el Grupo de Informática en Enfermería de la Universidad Federal de Sáo Paulo $^{(8,12)}$. Posteriormente, varias universidades brasileñas integraron la informática en enfermería en la informática de la salud y por lo tanto, las enfermeras tuvieron la oportunidad de interactuar con colegas multidisciplinarios ${ }^{(8,12)}$.

Los programas de enfermería del Instituto Universitario Argentino, vinculados al Hospital Italiano de Buenos Aires, incluyeron un curso de informática como parte del plan de estudios de enfermería ${ }^{(16)}$, con cuatro cursos (Informática básica I y II e Informática aplicada I y II). En el 2019, el Hospital Italiano de Buenos Aires organizó el primer Simposio de Informática en Enfermería, como parte de las XIV Jornadas sobre Sistemas 
de Información en Salud del Departamento de Informática en Salud del Hospital Italiano de Buenos Aires.

En Chile, hace casi una década, en la Universidad Central de Chile, Sede La Serena (Chile), se ofreció un diplomado en línea de informática en enfermería, dirigido a enfermeras que trabajaban en hospitales y programas comunitarios ${ }^{(10)}$. Además, la Pontificia Universidad Católica de Chile ofrece un Diplomado en Informática Clínica en línea dirigido a profesionales de la salud, incluyendo enfermeras (https://educacioncontinua.uc.cl/40 725-ficha-nuevo-diplomado-en-informaticaclinica). La Red Nacional de Enfermería Informática en Chile (REDENFI Chile) fue creada en 2013 y tiene un grupo activo de Facebook donde los miembros comparten seminarios web, cursos, artículos e información sobre informática de enfermería (https://www.facebook.com/groups/ 696562927659456/).

La enfermería informática en Cuba fue impulsada por la Red Nacional de Enfermería Informática (http://www.sld.cu/sitios/redenfermeria/), creada en 2010, donde técnicos y profesionales de enfermería cubanos cooperaron para el desarrollo de la informática de enfermería en el país ${ }^{(12)}$. En Colombia, la Red de Enfermería en Informática, denominada REDENFI Colombia, fue fundada en el año 2015 (http://www.enfermeriainformaticalac. org/index.php/redes/red-colombia).

La Escuela de Enfermería de la Universidad Michoacana de San Nicolás de Hidalgo de México, incluye en el plan de estudios: Informática y Teleenfermería (http://www.telenfermeria.umich. $\mathrm{mx} /$ ). En el grupo de Facebook titulado: "Red de Informática en Enfermería de México" (https:// www.facebook.com/riem2012?fref=ts) los miembros comparten noticias, eventos e información relacionada con la informática de enfermería.

Chérrez-Ojeda et al. ${ }^{(17)}$ reportaron que el $96,3 \%$ de las enfermeras ecuatorianas utilizaron las tecnologías de la información y comunicación (TIC) para comunicarse con sus colegas y el 80,1\% reportaron el uso de las TIC para comunicarse con sus pacientes. Más del $70 \%$ de los participantes estuvo de acuerdo en que las TIC pueden ser útiles para promover servicios profesionales, pueden ayudar a generar nuevas oportunidades laborales y/o desarrollo profesional, fomentar la promoción de la salud y mejorar el flujo de trabajo con sus colegas.

Finalmente, la informática en enfermería en Costa Rica se ha promovido desde principios de los 2000 y la incorporación de las tecnologías de la información y comunicación en el flujo de trabajo diario de la enfermería, en ese país, ha sido gradual pero constante ${ }^{(18)}$.

\section{LA NECESIDAD DE LOS PROGRAMAS DE INFORMÁTICA EN ENFERMERÍA EN EL PERÚ}

La enfermería representa la fuerza laboral más grande del sistema de salud peruano y el personal de enfermería es predominantemente femenino ${ }^{(19)}$. Para el año 2018, según el Colegio de Enfermeros del Perú, el 90\% de los profesionales de enfermería eran mujeres y el $10 \%$, hombres ${ }^{(19)}$. Se estima que por cada 10.000 habitantes en el sistema de salud peruano hay solo 12 enfermeras, y para cubrir la demanda en los hospitales se necesitarían urgentemente 250.000 enfermeras $^{(19)}$.

Las universidades peruanas que ofrecen cursos relacionados con la informática de la salud en programas de enfermería son limitadas. De acuerdo con Condor et al. ${ }^{(13)}$, de 62 programas relacionados con enfermería en universidades peruanas evaluadas, solo $15(24 \%)$ ofrecieron cursos específicos en informática de la salud, informática de enfermería o TIC para el cuidado de enfermería en su currículo académico de pregrado. También reportaron que un tercio de las universidades estaban ubicadas en Lima (34\%) y la mayoría eran privadas (67\%).

Por su parte, Uriarte ${ }^{(20)}$ informó la falta de voluntad y compromiso para la capacitación de algunas enfermeras que utilizaron un software de trazabilidad en un centro de esterilización de un hospital público de Chiclayo, Perú. Además, señaló que los resultados de su investigación servirían para revisar las competencias de enfermería con el fin de incluir las competencias de informática en enfermería ${ }^{(20)}$.

De igual forma, López e Ydrogo ${ }^{(21)}$ realizaron un estudio en Lambayeque (Perú), donde se evaluó el uso de las TIC en docentes de enfermería a nivel universitario. Participaron 120 personas de cuatro escuelas de enfermería y los autores encontraron que el $92 \%$ de los encuestados usaban computadora, el $90 \%$ contaban con una computadora portátil, 
93\% reportaron Internet en casa, 93\% usaban celular, $46 \%$ contaban con teléfonos inteligentes y 39\% usaban tabletas. Aunque se informó una buena penetración de las TIC en este grupo, el $58 \%$ de las personas informaron que no las usaban correctamente. Este estudio señaló que, a pesar del alto uso de las TIC en el grupo evaluado, se requería una formación adecuada para poder aprovechar al máximo la amplia gama de herramientas y usos de las TIC para la enfermería.

Estos resultados están alineados con la limitada literatura publicada sobre el uso de las TIC por parte del personal de enfermería, y en general del personal de salud, en el Perú ${ }^{(22)}$, sin embargo, algunos programas peruanos merecen ser destacados.

En Perú, la red Quipu, creada en 2010, tenía los siguientes objetivos: 1) desarrollar e implementar oportunidades de capacitación a corto y largo plazo en informática biomédica para la salud global en la Región Andina; 2) involucrar a nuevos investigadores en la Región Andina para que investiguen en informática en salud y bioinformática y 3) ampliar y consolidar una red de investigación en la Región Andina, promoviendo la colaboración Sur-Sur y las iniciativas colaborativas con universidades e instituciones de los Estados Unidos ${ }^{(9)}$. "Quipu" es una palabra quechua que describe un antiguo sistema utilizado en los Andes por los Incas para registrar y distribuir información ${ }^{(23)}$. El Centro ha implementado varios cursos presenciales y virtuales y ha apoyado el establecimiento de un diploma y un programa de maestría en Informática Biomédica, el primero de este tipo de programas de formación en el Perú( ${ }^{(9)}$ y los profesionales de enfermería fueron parte de la Red Quipu.

Se observa que es fundamental promover programas de investigación y capacitación en informática de enfermería, además de desarrollar redes de instituciones públicas y privadas con centros académicos que tengan experiencia en informática de enfermería.

En el año 2020, el Ministerio de Salud del Perú estableció las competencias básicas de enfermería para el manejo de las tecnologías de la información, comunicación y sistemas de información en salud, con énfasis en los ejes de desarrollo de la telesalud ${ }^{(24)}$. Este es un primer paso, muy importante, y sería recomendable tomar como referencia el perfil de competencias en informática de enfermería de la American Nurses Association y otros ${ }^{(5-9)}$. En Brasil, por ejemplo, el campo de las TIC es una competencia central desde 2001 y figura en las Directrices Curriculares Nacionales para cursos de pregrado en Enfermería $^{(8)}$. En este sentido, el enfermero debe "utilizar adecuadamente las nuevas tecnologías, tanto de la información como de la comunicación, como punta para el cuidado de la enfermería"(8). Según Staggers et al. ${ }^{(25)}$, las competencias validadas en informática en enfermería incluyen, entre otras, áreas de práctica como: educación, privacidad y seguridad, investigación, acceso y estructura de datos, gestión (de proyectos y financiera), mejora de la calidad, simulación, sistemas de selección y se incluyen habilidades para el uso de computadoras, conocimientos y habilidades informáticas.

Las universidades también deben trabajar en estrecha colaboración con los gobiernos locales, regionales y nacionales, para fomentar la informática en enfermería en el Perú( ${ }^{(9)}$. En este sentido, las alianzas con instituciones públicas y privadas, como parte de una estrategia bien planificada, han mostrado resultados alentadores ${ }^{(9)}$. Cada asociación es única y los programas de informática de enfermería deben garantizar que el plan de estudios sea relevante para abordar las necesidades locales y las prioridades del país ${ }^{(9)}$.

La promoción de la informática en enfermería puede incluir cursos, webinars, diplomados y maestrías impartidas por universidades, pero también el Colegio de Enfermeros del Perú puede jugar un papel importante en la promoción de la disciplina. De hecho, el Colegio de Enfermeros del Perú actualmente ofrece webinars, brindando oportunidades para adquirir conocimientos y habilidades en diferentes temas (https://www. facebook.com/cep.org.pe/). Además, los programas de capacitación en informática en enfermería en el Perú deben promover las competencias de liderazgo y fomentar la exposición internacional y pasantías en instituciones con experiencia relevante en informática en enfermería en América Latina y el mundo.

De igual forma, el grupo de expertos en salud digital, establecido por la Organización Mundial de la Salud, puede ser un mecanismo para impulsar la colaboración entre expertos en informática médica y de enfermería de América Latina y el extranjero $^{(9)}$. Para ello, es fundamental compartir las experiencias y lecciones aprendidas con respecto a la implementación y evaluación de programas de 
desarrollo de capacidades para la informática en enfermería.

\section{DESAFÍOS PARA LOS PROGRAMAS DE CAPACITACIÓN EN INFORMÁTICA EN ENFERMERÍA EN EL PERÚ}

Es clave que las enfermeras peruanas reconozcan la necesidad de documentar su práctica de enfermería de manera completa. Las historias clínicas electrónicas y la telesalud aún no se han implementado ampliamente en Perú debido a desafíos organizativos, físicos y tecnológicos ${ }^{(9)}$. Sin embargo, las enfermeras deben participar estrecha y activamente en el diseño y la implementación de los sistemas de información de salud y en cómo se desarrollan dichos sistemas para registrar los datos de enfermería ${ }^{(5-9,26)}$.

Las enfermeras peruanas deberían estar más conectadas y las redes sociales podrían desempeñar un papel orientado a compartir información y recursos sobre informática de enfermería ${ }^{(9)}$. En Perú existe un grupo en Facebook denominado "Red Peruana de Enfermería Informática" (https:// www.facebook.com/redperuei/) que promueve la investigación, los webinars y el debate sobre el uso de las tecnologías de la información y la comunicación para apoyar la atención de enfermería. Dado que tenemos una escasez de informáticos en enfermería en el Perú, es importante promover cursos y programas virtuales sobre informática en enfermería.

Otro desafío es cómo expandir los poquísimos cursos de informática de enfermería en todo el Perú, promoviendo una colaboración efectiva a nivel nacional e internacional en la configuración del currículo académico y la agenda de investigación. Por lo tanto, el rol de las alianzas colaborativas podría ser muy útil para compartir recursos, experiencias y lecciones aprendidas entre países, para optimizar la capacitación en informática en enfermería y oportunidades de investigación en América Latina.

La Red Internacional de Enfermería Informática (http://www.enfermeriainformaticalac.org/), creada en 2008, fue patrocinada inicialmente por la Organización Panamericana de la Salud con participación de técnicos y profesionales de enfermería de países como Argentina, Brasil,
Chile, Colombia, Cuba, El Salvador, México, Perú, España, Uruguay y Venezuela. Esta red podría desempeñar un papel importante para apoyar la creación de capacidad de recursos digitales sobre salud, el intercambio de tecnologías de la información y promover proyectos de investigación conjuntos.

La Federación de Informática en Salud para América Latina y el Caribe (IMIA LAC) Grupo de Enfermería Informática (https://www.facebook. com/wgniimialac.enfermeria) ha organizado talleres de capacitación para enfermeras para mejorar sus habilidades en el uso de las TIC en el punto de atención. Además, el Grupo de Informática de Enfermería IMIA LAC desarrolló reuniones virtuales y teleconferencias durante los últimos años sobre una diversidad de temas como: bibliotecas virtuales, programación de computadoras, y TIC y Educación en Enfermería.

Finalmente, es importante reexaminar lo que esperamos que las enfermeras peruanas sepan sobre temas de privacidad, confidencialidad y seguridad en un ecosistema de salud digital ${ }^{(9)}$. Pueden surgir nuevos factores legales, éticos, sociales y de política pública, considerando los cambios socio-legales y $\operatorname{políticos}^{(9)}$.

\section{DESARROLLO DE CAPACIDADES EN TIEMPOS DE COVID-19}

La pandemia por el COVID-19 exige una acción urgente y necesaria para transformar la prestación de los servicios de salud, para expandir los programas e iniciativas de teleenfermería y de enfermería a distancia en todo el mundo ${ }^{(27)}$. Específicamente, la telesalud se utilizó para monitorear el estado de salud de las poblaciones y disminuir el número de personas que acceden a los centros de salud locales, con la participación de muchos enfermeros informáticos durante el proceso ${ }^{(28)}$.

Es importante señalar que, además de la pandemia por el COVID-19, la enfermería informática es parte integral de todas las acciones de emergencia que toman las organizaciones de salud durante desastres naturales, conflictos sociales y emergencias nacionales ${ }^{(29)}$.

Dada la creciente prevalencia del COVID-19 en Perú, los centros de salud carecen de enfermeras especializadas y existe un grave déficit de unidades 
de cuidados intensivos para pacientes con COVID-19 ${ }^{(30)}$. Por tanto, la teleenfermería parece una tremenda oportunidad en la crisis pandémica actual, que permitiría mejorar la respuesta asistencial y la calidad de la atención ${ }^{(31)}$.

\section{CONCLUSIÓN}

Es necesario estandarizar la formación de enfermería en el Perú, incluyendo la definición de perfiles de competencia en informática aplicada a la enfermería, de acuerdo con las necesidades de salud del país y considerando los factores económicos, sociales, culturales, legales, organizacionales y los desafíos globales. En el Perú se debe impulsar la creación y fortalecimiento de las redes existentes de instituciones públicas y privadas con centros académicos que tienen experiencia en formación e

\section{REFERENCIAS}

1. World Health Organization. Nursing and midwifery [Internet]. Geneva, Switzerland 2020 jan 9 [citado 22 ene 2021]. Disponible en: https://www. who.int/news-room/fact-sheets/detail/nursingand-midwifery

2. Rosa WE, Dossey BM, Koithan M, Kreitzer MJ, Manjrekar P, Meleis AI, et al. Nursing Theory in the Quest for the Sustainable Development Goals. Nurs Sci Q [Internet]. 2020 [citado 22 ene 2021]; 33(2):178-182. Disponible en: $10.1177 / 0894318420903495$.

3. Forman TM, Armor DA, Miller AS. A Review of Clinical Informatics Competencies in Nursing to Inform Best Practices in Education and Nurse Faculty Development. Nurs Educ Perspect [Internet]. 2020 [citado 22 ene 2021]; 41(1): E3-E7. Disponible en: 10.1097/01.NEP.000000 0000000588

4. Topaz M, Ronquillo C, Peltonen LM, Pruinelli L, Sarmiento RF, Badger MK, et al. Advancing Nursing Informatics in the Next Decade: Recommendations from an International Survey. Stud Health Technol Inform [Internet]. 2016 [citado 22 ene 2021]; 225: 123-7. Disponible en: 10.3233/978-1-61499-658-3-123 investigación en informática en enfermería.

Todos los profesionales de enfermería tienen un rol en la informática, y ahora es el momento de acelerar el ritmo para desarrollar la informática de enfermería y las habilidades de liderazgo, pero siempre considerando un enfoque centrado en la persona. Las enfermeras deben aprovechar esta oportunidad para adaptar la tecnología a la práctica de enfermería, en lugar de adaptar la práctica de enfermería a la tecnología. Los programas de capacitación en enfermería informática en el Perú deben incorporar el contexto local, considerar sus necesidades y deben ser sensibles a los factores económicos, sociales, culturales y organizativos locales. Por último, el liderazgo en informática de enfermería es fundamental en todos los niveles y en todos los entornos clínicos, administrativos, educativos y de investigación, especialmente durante la pandemia de COVID-19 $9^{(32)}$.

5. American Nurses Association (ANA). Nursing Informatics: Scope and Standards of Practice [Internet]. $2^{\text {nd }}$ ed. United States. 2014 [citado 22 ene 2021]. 235 p. Disponible en: https:// www.nursingworld.org/nurses-books/nursinginformatics-scope-and-standards-of-practice-2nded/

6. Honey ML, Skiba DJ, Procter P, Foster J, Kouri P, Nagle LM. Nursing Informatics Competencies for Entry to Practice: The Perspective of Six Countries. Stud Health Technol Inform [Internet]. 2017 [citado 22 ene 2021]; 232:51-61. Disponible en: 10.3233/978-1-61499-738-2-51

7. Strudwick G, Nagle L, Kassam I, Pahwa M, Sequeira L. Informatics Competencies for Nurse Leaders: A Scoping Review. J Nurs Adm [Internet]. 2019 [citado 22 ene 2021]; 49(6): 323-330. Disponible en: $10.1097 /$ NNA.0000000000000760.

8. Barbosa SF. Competencies Related to Informatics and Information Management for Practicing Nurses and Nurses Leaders in Brazil and South America. Stud Health Technol Inform [Internet]. 2017 [citado 22 ene 2021]; 232:77-85. Disponible en: 10.3233/978-1-61499-738-2-77

9. Curioso WH. Building Capacity and Training for Digital Health: Challenges and Opportunities in Latin America. J Med Internet Res [Internet]. 2019 
[citado 22 ene 2021]; 21(12):e16513. Disponible en: $10.2196 / 16513$.

10. Hernández CA, Wigodski SJ, Caballero ME. Informática en enfermería un desafío a la formación actual. Medwave [Internet]. 2012 [citado 22 ene 2021]; 2: 1-4. Disponible en: 10.5867/ medwave.20012.02.5307.

11. Hullin C. Nursing Informatics Education: Latino America \& Caribe. Stud Health Technol Inform [Internet]. 2016 [citado 22 ene 2021]; 225:72931. Disponible en: https://europepmc.org/article/ $\mathrm{med} / 27332321$

12. Vialart VN. Apuntes y experiencias en el desarrollo de la informática en Enfermería de Cuba. Rev Cubana Enfermer [Internet]. 2007 [citado 22 ene 2021]; 23(3): 1-11. Disponible en: http://scielo. sld.cu/scielo.php?script=sci_arttext\&pid=s086403192007000300003

13. Condor DF, Sánchez AK, Bidman AA. Nursing Informatics Training in Undergraduate Nursing Programs in Peru. Studies in Health Technology and Informatics [Internet]. 2018 [citado 22 ene 2021]; 250:81. Disponible en: 10.3233/978-161499-872-3-81

14. Marín HF, Silveira DT, Sasso GD, Pérez HHC. Evolution: Nursing Informatics in Brazil. In: Ball M. et al. (eds.) Nursing Informatics. Health Informatics [Internet]. London: Springer; 2012 [citado 22 ene 2021]: 401-410. Disponible en: https://link.springer.com/chapter/10.1007/978-184996-278-0_24

15. Otero P, Leikam M, González Z, Marín HF, Aravena IP, Zawadzki S. Informatics Education in Latin America. In: Berner ES (ed). Informatics education in Healthcare. Health Informatics [Internet]. Cham: Springer; 2020 [citado 22 ene 2021]: 167182. Disponible en: https://link.springer.com/ chapter/10.1007\%2F978-3-030-53813-2_13

16. González ZA, Schachner MB, Tattone MA, Benítez SE. Changing Educational Paths in an Informatics Course According to the Needs and Expectations of Nursing Degree Students. Stud Health Technol Inform [Internet]. 2016 [citado 22 ene 2021]; 225:324-8. Disponible en: https://europepmc.org/ article/med $/ 27332215$

17. Chérrez-Ojeda I, Felix M, Valeria L, Mata VE, Simancas-Racines D, Aguilar M, et al. Use and Perceptions of Information and Communication Technologies Among Ecuadorian Nurses: A Cross-sectional Study. The Open Nursing Journal [Internet]. 2020 [citado 22 ene 2021]; 14: 8-17. Disponible en: 10.2174/1874434602014010008.

18. González-Sáenz G. Evolución histórica de la enfermería informática en Costa Rica. Enfermería en Costa Rica [Internet]. 2007 [citado 22 ene
2021]; 28(1): 26-28. Disponible en: http://www. binasss.sa.cr/revistas/enfermeria/v28n1/art5.pdf

19. Andina. Más de 89,000 profesionales de la enfermería celebran su día en el Perú [Internet]. Lima: Agencia Nacional de Noticias (C) 2017; Mayo 12, 2018 [citado 22 ene 2021]. Disponible en: https://andina.pe/agencia/noticia-mas-89-milprofesionales-de-enfermeria-celebran-su-dia-elperu-709747.aspx

20. Uriarte BZ. Percepciones del personal de enfermería en el proceso de implementación del programa de trazabilidad. Central de esterilización. Hospital III-1, MINSA. Chiclayo, 2017 [Tesis] [Internet]. Chiclayo: Universidad Católica Santo Toribio de Mogrovejo; 2018 [citado 22 ene 2021]. Disponible en: http://54.165.197.99/ bitstream/20.500.12423/1294/1/TL_UriarteBecerra Zulema.pdf.pdf

21. López RCJ, Ydrogo SJR. Uso de tecnologías de la información y la comunicación (TIC) por parte de enfermeros docentes en la región Lambayeque, 2017 [Tesis] [Internet]. Chiclayo: Universidad Católica Santo Toribio de Mogrovejo; 2018 [citado 22 ene 2021]. Disponible en: http://tesis.usat.edu. pe/handle/20.500.12423/1293

22. González-Argote J. La producción científica latinoamericana sobre historia clínica digital: un análisis desde Scopus. Rev Cubana Salud Pública [Internet]. 2019 [citado 22 ene 2021]; 45(3): e1312. Disponible en: http://www.revsaludpublica. sld.cu/index.php/spu/article/view/1312/1386

23. Blas MM, Curioso WH, García PJ, Zimic M, Cárcamo CP, Castagnetto JM, et al. Training the biomedical informatics workforce in Latin America: results of a needs assessment. BMJ Open [Internet]. 2011 Jan [citado 22 ene 2021]; 1(2): e000233. Disponible en: 10.1136/bmjopen-2011-000233.

24. Ministerio de Salud. Resolución Ministerial $\mathrm{N}^{\circ}$ 960-2020-MINSA. Perfil de competencias esenciales que orientan la formación de los profesionales de la salud, Primera fase: Médico(a) y Enfermero(a) peruano(a) [Internet]. Lima, Perú. 2020 [citado 22 ene 2021]. 66 p. Disponible en: https://www.gob.pe/institucion/minsa/normaslegales/1364189-960-2020-minsa

25. Staggers N, Gassert CA, Curran C. A Delphi study to determine informatics competencies for nurses at four levels of practice. Nurs Res [Internet]. 2002 Nov-Dec [citado 22 ene 2021]; 51(6): 38390. Disponible en: https://journals.lww.com/ nursingresearchonline/Fulltext/2002/11000/A _Delphi_Study_to_Determine_Informatics.6. aspx?casa_token=ccNfoMYdwhQAAAAA:YS_lod Oz5cWttpeeSk2t40vqWiMsbG2dQ8wkwnhxg3 xb02ZeRsCSLHssCzGMsmRKuUa7AbdiGMM- 
y6UVefK6WpgBlb05

26. Mantas J, Ammenwerth E, Demiris G, Hasman A, Haux R, Hersh W, et al. Recommendations of the International Medical Informatics Association (IMIA) on Education in Biomedical and Health Informatics. First Revision. Methods Inf Med [Internet]. 2010 [citado 22 ene 2021]; 49(2): 105-120. Disponible en: 10.3414/ME5119.

27. Atique S, Bautista JR, Block LJ, Lee JJ, LozadaPerezmitre E, Nibber R, et al. A nursing informatics response to COVID-19: Perspectives from five regions of the world. J Adv Nurs [Internet]. 2020 Oct [citado 22 ene 2021]; 76(10): 2462-2468. Disponible en: $10.1111 /$ jan.14417.

28. Sood A, Pollard C, Suer KL, Vlahovich K, Walker J. Caring for Miners During the Coronavirus Disease-2019 (COVID-19) Pandemic. J Rural Health [Internet]. Epub 2020 Jun 2 [citado 22 ene 2021]; 37(1): 165-168. Disponible en: 10.1111/ jrh.12444.

29. Nejadshafiee $M$, Bahaadinbeigy K, Kazemi $M$, Nekoei-Moghadam M. Telenursing in Incidents and Disasters: A Systematic Review of the Literature. J Emerg Nurs [Internet]. 2020 Sep [citado 22 ene 2021]; 46(5): 611-622. Disponible en: 10.1016/j.jen.2020.03.005.

30. Acosta G, Escobar G, Bernaola G, Alfaro J, Taype W, Marcos C, et al. Caracterización de pacientes con COVID-19 grave atendidos en un hospital de referencia nacional del Perú. Rev Peru Med Exp Salud Pública [Internet]. 2020 [citado 22 ene 2021]; 37(2): 253-8. Disponible en: 10.17843/ rpmesp.2020.372.5437

31. Watkins S, Neubrander J. Primary-care registered nurse telehealth policy implications. J Telemed Telecare [Internet]. 2020 Aug [citado 22 ene 2021]; 3: 1357633X20940142. Disponible en: 10.1177/1357633X20940142.

32. Hoffmann RL, Battaglia A, Perpetua Z, Wojtaszek K, Campbell G. The Clinical Nurse Leader and COVID-19: Leadership and quality at the point of care. J Prof Nurs [Internet]. 2020 Jul-Aug [citado 22 ene 2021]; 36(4): 178-180. Disponible en: 10.1016/j.profnurs.2020.06.008. 